\title{
Consensus Statements by Korean Society of Interventional Neuroradiology and Korean Stroke Society: Hyperacute Endovascular Treatment Workflow to Reduce Door-to-Reperfusion Time
}

Dae-Hyun Kim, MD, PhD ${ }^{1 *}$, Byungjun Kim, MD, PhD ${ }^{2 *}$, Cheolkyu Jung, MD, PhD ${ }^{3}$, Hyo Suk Nam, MD, PhD ${ }^{4}$, Jin Soo Lee, MD, PhD ${ }^{5}$, Jin Woo Kim, MD ${ }^{6}$, Woong Jae Lee, MD, PhD ${ }^{7}$, Woo-Keun Seo, MD, PhD', Ji-Hoe Heo, MD, PhD ${ }^{4}$, Seung Kug Baik, MD, PhD ${ }^{9}$, Byung Moon Kim, MD, PhD ${ }^{10}$, Joung-Ho Rha, MD, PhD ${ }^{11}$

${ }^{1}$ Department of Neurology, Dong-A University Hospital, Busan 49201, Korea; ${ }^{2}$ Department of Radiology, Korea University Anam Hospital, Seoul 02841, Korea; ${ }^{3}$ Department of Radiology, Seoul National University Bundang Hospital, Seongnam 13620, Korea; Departments of ${ }^{4}$ Neurology and ${ }^{10}$ Radiology, Yonsei University Severance Hospital, Seoul 03722, Korea; ${ }^{5}$ Department of Neurology, Ajou University School of Medicine, Suwon 16499, Korea; ${ }^{6}$ Department of Radiology, Inje Univeristy Ilsan Paik Hospital, Goyang 10380, Korea; ${ }^{7}$ Department of Radiology, Chung-Ang University Hospital, Seoul 06973, Korea; ${ }^{8}$ Department of Neurology, Sungkyunkwan University, Samsung Medical Center, Seoul 06351, Korea; ${ }^{9}$ Department of Radiology, Pusan National University Yangsan Hospital, Yangsan 50612, Korea; ${ }^{11}$ Department of Neurology, Inha University Hospital, Incheon 22332, Korea

Recent clinical trials demonstrated the clinical benefit of endovascular treatment (EVT) in patients with acute ischemic stroke due to large vessel occlusion. These trials confirmed that good outcome after EVT depends on the time interval from symptom onset to reperfusion and that in-hospital delay leads to poor clinical outcome. However, there has been no universally accepted in-hospital workflow and performance benchmark for rapid reperfusion. Additionally, wide variety in workflow for EVT is present between each stroke centers. In this consensus statement, Korean Society of Interventional Neuroradiology and Korean Stroke Society Joint Task Force Team propose a standard workflow to reduce door-to-reperfusion time for stroke patients eligible for EVT. This includes early stroke identification and pre-hospital notification to stroke team of receiving hospital in pre-hospital phase, the transfer of stroke patients from door of the emergency department to computed tomography (CT) room, warming call to neurointervention (NI) team for EVT candidate prior to imaging, NI team preparation in parallel with thrombolysis, direct transportation from CT room to angiography suite following immediate decision of EVT and standardized procedure for rapid reperfusion. Implementation of optimized workflow will improve stroke time process metrics and clinical outcome of the patient treated with EVT.

Keywords: Endovascular treatment; Door-to-reperfusion time; Hyperacute stroke; Pre-hospital notification

Received June 6, 2018; accepted after revision June 6, 2018.

This article is the secondary publication of the following article entitled "Consensus Statements by Korean Society of Interventional Neuroradiology and Korean Stroke Society: Hyperacute Endovascular Treatment Workflow to Reduce Door-to-Reperfusion Time," which was published in the Journal of Korean Medical Science (JKMS) Volume 33 (19), May 2018. This article has been published jointly by invitation and consent in both the Korean Journal of Radiology (KJR) and the JKMS. The JKMS and KJR officially agree with the authors' requests that the KJR be able to issue a secondary publication in order to disseminate important information to the widest possible audiences.

*These authors contributed equally to this work.

Corresponding author: Joung-Ho Rha, MD, PhD, Department of Neurology, Inha University Hospital, Inha University College of Medicine, 27 Inhang-ro, Jung-gu, Incheon 22332, Korea.

- Tel: (8232) 890-3668• Fax: (8232) 890-3864•E-mail: jhrha@inha.ac.kr; and

Byung Moon Kim, MD, PhD, Interventional Neuroradiology, Severance Hospital Stroke Center, Department of Radiology, Yonsei University College of Medicine, 50-1 Yonsei-ro, Seodaemun-gu, Seoul 03722, Korea.

- Tel: (822) 2228-2399 • Fax: (822) 393-3035•E-mail: bmoon21@hanmail.net

This is an Open Access article distributed under the terms of the Creative Commons Attribution Non-Commercial License (https:// creativecommons.org/licenses/by-nc/4.0) which permits unrestricted non-commercial use, distribution, and reproduction in any medium, provided the original work is properly cited. 


\section{INTRODUCTION}

Endovascular treatment (EVT) has been proven to be beneficial for acute ischemic stroke patients with a large vessel occlusion, and its efficacy is highly timedependent (1). The key to a good outcome is short onsetto-eperfusion time, and accelerating each step from prehospital notification to reperfusion significantly contributes to an overall improved outcome. Among those steps, door (hospital arrival)-to-puncture time can be significantly reduced by a standardized multidisciplinary approach $(2,3)$. Therefore, the Korean Society of Interventional Neuroradiology and Korean Stroke Society collaborated to make a joint task force team to make consensus statement of hyperacute EVT workflow for implementation and dissemination to acute stroke care hospitals.

\section{Pre-Hospital Phase}

Pre-hospital stroke recognition by providers of emergency medical services (EMS) is an initial and critical step to reduce the delay in early treatment. Pre-hospital stroke scales allow EMS personnel to identify acute stroke patients with high degree of sensitivity (78-95\%) and specificity (56-95\%) (4). It is recommended that EMS personnel use a standardized pre-hospital stroke screening tool, such as the Los Angeles Prehospital Stroke Screen or Cincinnati Prehospital Stroke Scale (5-7). Regular education and feedback to EMS personnel would be necessary to improve their skills in recognizing the signs and symptoms of stroke $(8,9)$. It would be beneficial to further develop screening tools to predict large artery occlusion-related stroke, and to validate in the Korean population.

Pre-hospital notification of stroke patient arrival by EMS paramedics reduces in-hospital delay because it facilitates early activation of stroke team and preparation of imaging modalities prior to patient arrival (10-14). Additionally, pre-hospital notification allows stroke team members to be prepared at the emergency room prior to the arrival of the patient. The Get With the Guidelines-Stroke program found reduced door-to-imaging, door-to-needle, and onsetto-needle time in patients with pre-hospital notification compared with those in patients without pre-hospital notification (10). Despite these benefits, EMS pre-hospital notification was found to occur in only two-thirds of patients with acute ischemic stroke arriving by EMS in the United States (15). It is generally assumed that the rate of pre-hospital notification is lower in Korea than in the United States $(12,16)$.

\section{Consensus Statements}

- It is recommended that EMS personnel are to be trained to rapidly identify suspected stroke patients using a standardized pre-hospital stroke screening tool.

- It is recommended that EMS personnel make prehospital notification calls to the receiving hospital to allow for the activation of the stroke team prior to the patient's arrival.

\section{In-Hospital Pre-Angiography Suite Phase}

\section{Approach for Rapid Door-to-Imaging Time on Arrival}

Upon arrival to the emergency department, suspected stroke patients should be assigned a hospital identification number with high priority at the triage desk for subsequent diagnostic or treatment orders. Reducing hospital delay requires rapid triage, notification, and action by many medical staff members in various departments, including the emergency, neurology, nursing, neuroimaging, and laboratory departments. Single-call systems to activate all stroke team members can reduce in-hospital processing time in patients with acute ischemic stroke. A computerized in-hospital alert system using a computerized physician order entry has been developed and was demonstrated to be effective $(13,17)$.

The pre-notification of possible EVT candidates can be given to the neurointervention (NI) team as a "warming call" prior to image acquisition. This enables NI team members to prepare for possible endovascular thrombectomy. According to a survey of stroke centers in the United States, initial contact with an NI physician was made prior to image acquisition at $86 \%(25 / 29)$ of centers (18). Direct contact information of NI on-duty members is regularly updated and announced to stroke team members. In hyperacute stroke patient suspected of large-vessel occlusions (National Institutes of Health Stroke Scale [NIHSS] $\geq 10$ ), a warning call to the NI team by the stroke physician is encouraged prior to the acquisition of additional vascular imaging. However, the use of the NIHSS as a surrogate marker to identify large-vessel occlusion may result in a risk of missing $30 \%$ of anterior circulation strokes and $60 \%$ of posterior circulation strokes (19). Considering the inaccuracy of NIHSS in predicting largevessel occlusion, a prompt acquisition of non-invasive 
vascular imaging is highly recommended in patients with low NIHSS to confirm large vessel disease (20).

Radiologic technologist is to ensure the availability of the computed tomography (CT) scanner immediately following the activation of a stroke team. In the emergency room, it is recommended that the patient is moved directly from the door to the CT scanner (2, 21). A retrospective review of stroke patients receiving intravenous recombinant tissue plasminogen activator ( $r$-tPA) reported that EKG performance and chest $X$-rays prior to the brain CT scan increased door-to-imaging time by 6 and 13 minutes, respectively (22). In case of default CT scanner unavailability, a secondary CT or MRI scanner can be used (21). Focused clinical assessment, recording of patient history, NIHSS assessment, and blood sampling for laboratory evaluation can be performed simultaneously in the CT room $(23,24)$.

\section{Consensus Statements}

- It is recommended that, upon pre-hospital notification or arrival of stroke-suspected patient, all stroke team members are activated by a single call.

- The notification call to the stroke team can be given to the NI team as a "warming call" for preparation for a possible EVT candidate.

- The suspected stroke patient can be transported directly from the door to the CT scanner for rapid clinical assessment and blood sampling without delay.

\section{Brain Imaging}

Noncontrast CT is the initial imaging scan to determine intravenous r-tPA eligibility, and can be immediately followed by CT angiography (CTA) in order to decide an endovascular approach. CTA rapidly and noninvasively provides important information about vessel occlusion or stenosis of intracranial and extracranial vasculature (25). Current stroke guidelines recommend CT/CTA to be an initial evaluation of possible acute stroke patient (26-28). If magnetic resonance (MR) protocol is feasible and can be performed with similar time interval compared with $\mathrm{CT}$ at certain hospitals, MR angiography (MRA) can be used instead. In limited cases, such as uncertain stroke or stroke mimics, unclear-onset stroke, or posterior circulation stroke, additional imaging such as CT perfusion (CTP) or multimodal MR could be utilized to increase the accuracy of diagnosis and assess the risk/benefit of aggressive treatment. Additionally, these imaging protocols should not delay r-tPA infusion or EVT $(7,27)$.

\section{Consensus Statement}

- Computed tomography and CTA are recommended as the initial vascular imaging method for consideration of urgent EVT in acute stroke patient, and additional imaging should not delay r-tPA and EVT.

\section{Safety Concern of Contrast for CTA and EVT \\ Computed tomography angiography is becoming} increasingly important to rapidly identify large artery occlusion for possible EVT in patients with acute ischemic stroke. Because of the potential risk of contrast-induced acute kidney injury, serum creatinine levels are usually evaluated before performing CTA. However, CTA is occasionally delayed due to awaiting the laboratory results of serum creatinine level or estimated glomerular filtration rate. Many previous studies have reported low incidence (2-4\%) of contrastinduced nephropathy (> 25\% increase in creatinine) in acute stroke patients who underwent emergency multimodal CT scanning or an emergency endovascular intervention, which does not significantly differ from the other conditions (29, 30). It can be debatable whether or not it is justifiable to perform CTA without a baseline serum creatinine in hyperacute ischemic stroke (29). However, considering the rapid neuronal death rate (2 million per minute) and the resulting disability of patient, the harm caused by delayed treatment in possible stroke patients outweighs the benefit of preventing low incidence, and mostly reversible, renal injury. Thus, in cases of possible stroke with no history or clinical evidence of renal impairment or severe dehydration, doctors need not to await the laboratory results prior to commencing CTA/CTP (27).

\section{Consensus Statement}

- In cases with no history or clinical evidence of previous kidney disease or severe dehydration, CTA or CTP does not have to be delayed in suspected hyperacute stroke patients due to concern of possible renal injury by contrast agent.

\section{Obtaining Informed Consent}

Informed consent provided by the patient or relatives is required by medical ethics and law prior to initiating potentially harmful therapy. However, in cases of hyperacute stroke, patients typically lack the capacity to provide consent due to impaired consciousness, aphasia, or other neurological deficits. Relatives are frequently 
absent; therefore, obtaining informed consent could be the "rate-limiting step" and might delay the treatment with evidence of high efficacy. The Canadian Stroke Network Study reported that the acquisition rate of informed consent was as low as $39 \%$ in hyperacute stroke treatment and recommended waivers of informed consent (31). In concordance with these findings, it was found that certain stroke centers surpassed the formal consent process for EVT in the hospital workflow (21).

In Korea, the law for EMS regulates that if a physician is required to treat an urgent patient without decisionmaking capacity, and cannot receive consent from a legal representative or proxy, the physician may treat the patient following acquisition of documented consent provided by another physician based on medical judgement (32). Individual institutions must establish respective protocols for this situation. In addition, informed consent for conscious sedation (CS) can be acquired while obtaining informed consent for EVT, allowing for rapid implementation of CS.

\section{Consensus Statements}

- Obtaining informed consent should not delay the time to commence urgent EVT.

- In the event that hyperacute patients are unable to provide consent due to neurological deficit, and legal representative is unavailable, the physician may perform EVT following acquisition of documented consent provided by another physician.

\section{Procedures for Bridging r-tPA Administration}

Intravenous $\mathrm{r}$-tPA can be administered in the $\mathrm{CT}$ room immediately after the stroke physician has determined whether the noncontrast CT finding is compatible with intravenous thrombolysis eligibility (2). If the emergency room stroke kit, which contains necessary materials such as the informed consent sheet and r-tPA agents (Fig. 1), is placed at the designated location, the stroke team can avoid unnecessary time delay for preparation (21). Following intravenous r-tPA, waiting for patient observation to evaluate the clinical response prior to pursuing EVT is not necessary (26).

\section{Consensus Statements}

- It is recommended that intravenous r-tPA is administered in the CT room immediately following decision making, and patient observation to evaluate the clinical response is not necessary prior to EVT.

- Storing the stroke kit in the emergency room can be advantageous for rapid administration of $r$-tPA following determination of the treatment strategy.

\section{Optimal Workflow from Imaging to the Angiography Suite}

Within the past year, secondary analyses of clinical trials have established that achieving a reduced imagingto-reperfusion time significantly improved the chance of achieving a functionally independent outcome (33-35). The Endovascular Treatment for Small Core and Proximal

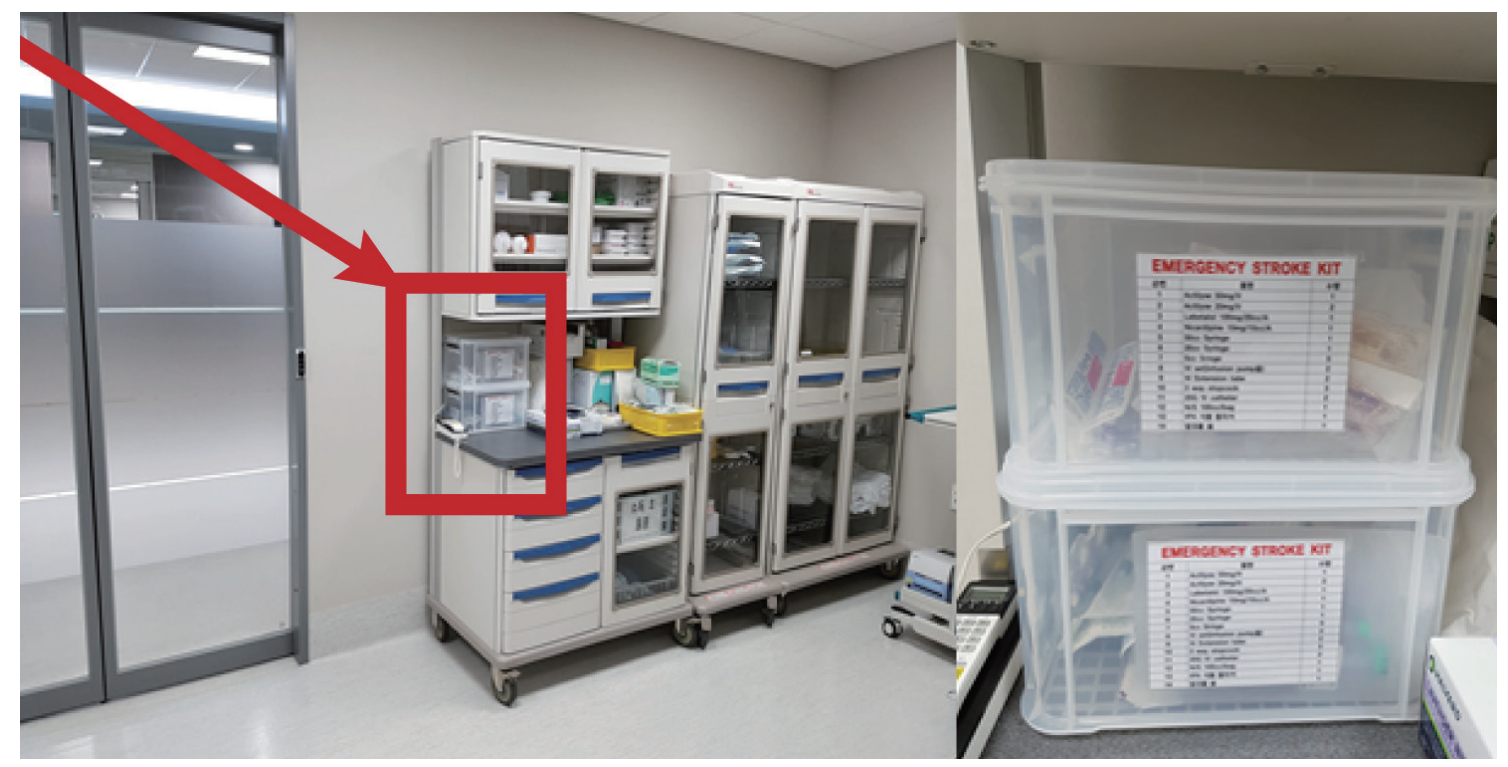

Fig. 1. Customized emergency stroke kit. Kit contains basic equipment for IV tPA administration, such as Actylase, Labetolol, Nicardipine, syringes, IV catheter, normal saline bag, and informed consent. IV tPA = intravenous tissue plasminogen activator 
Occlusion Ischemic Stroke (ESCAPE) trial placed emphasis on the swiftness of image acquisition and interpretation, transfer to the angiography suite, and endovascular techniques (36). Considering the fact that the imagingto-puncture metric reflects inefficiency across specialties and geographic space, multidisciplinary coordination is imperative to achieve highly efficient workflow metrics.

Imaging time is defined as the acquisition time of the first slice of noncontrast brain CT scan (35). This definition encompasses the acquisition and interpretation of optimal brain imaging to rapidly determine the need for thrombolytic therapy and intraarterial mechanical thrombectomy. Therefore, establishing the optimal brain imaging method for a patient who will benefit from EVT is a key initial step to reduce the inefficiencies in workflow that delay the delivery of treatment.

Early activation of the NI team is highly recommended following rapid determination of EVT by stroke team neurologists after identification of large-vessel occlusion on non-invasive brain vascular imaging (CTA or MRA) $(23,37)$. Marked improvements have been observed in a pilot quality improvement project, after early activation of the NI team led to a decrease in door-to-puncture time from a median of 143 minutes to a mean of 107 minutes (3).

The "no turn back" approach to the emergency room following brain imaging is a viable option to reduce doorto-puncture and image-to-puncture time in acute ischemic stroke patients undergoing EVT. Qureshi et al. (38) reported a significantly higher rate of $\mathrm{CT}$ scan acquisition to a microcatheter placement time of less than 90 minutes in patients in whom the no turn back approach was used compared to patients in whom this approach was not used $(57.6 \%$ vs. $31.6 \%, p<0.001)$. Thus, with intravenous $r$-tPA administration, patients who are eligible for endovascular thrombectomy should be directly transported to the angiographic suite without returning to the emergency room $(2,38)$.

\section{Consensus Statements}

- A standardized multidisciplinary approach is imperative to improve efficiency in imaging-to-puncture workflow.

- Establishment of optimal image protocol is a key initial step to identify patients who are eligible for EVT. Rapid image acquisition and interpretation should be followed to increase the efficiency of the imaging-to-puncture process.

- Early activation of the NI team by stroke physicians is recommended to minimize delays in preparation of the angiography suite.

- Patients eligible for EVT should be directly transported from the $\mathrm{CT}$ room to the angiographic suite without returning to the emergency room.

\section{In-Hospital Angiography Suite Phase}

\section{Preparing EVT in the Angiography Suite Prior to Arrival}

Following on-call NI team activation, a parallel workflow is initiated according to the pre-assigned roles of the team members. Essential members of the NI team generally consist of an NI physician, assistant NI physician, exclusive charge nurse of the angiography suite, and an NI technologist. In addition, collaborative members are designated to prevent contingencies. The NI physician evaluates non-invasive vascular imaging containing information regarding the craniocervical vasculature of the patient and determines a strategy of an endovascular approach considering the anatomy of the aortic arch and supraaortic large vessels (39). In parallel, the nurse and NI technologist prepare the angiography suite for the upcoming thrombectomy procedure (40). This team-based approach resulted in a marked reduction in the treatment time and increase in the number of patients successfully treated in the American Heart Association's Best Practice Strategies (41).

Optimal organization of on-call NI team members is crucial to resolve imbalances in delivery of care between office hours and after-hours. The imaging-to-puncture time is increased during after-hours than during office hours (42). Several studies have raised the factor of patient presentation during office hours as an independent predictor for reduced door-to-puncture times $(3,43,44)$. The time spent moving the NI team to the hospital after calling is a critical time-limiting step on weekends or during nights in many institutions. To reduce the time delay during the after-hour period, it would be advantageous if appropriate compensation should follow to endovascular paramedics at each intervention case. To maintain 24-hour/7-day coverage of EVT, a minimum of two NI teams are required to rotate on duty. Maintaining a more consistent level of activity at night or during weekends is often economical, despite higher wages for weekend duties (45). Additionally, motivation of NI personnel via appropriate feedback is essential. Every member of the NI team should be aware of the impact of acute intervention on the stroke patient's life and is required to provide a follow-up report of individual patients' status. 


\section{Consensus Statements}

- It is highly recommended that the NI team is wellorganized and is announced to the emergency department and stroke physicians. Following NI team activation, emphasis is placed on parallel processing of tasks and participation of all team members in favor of immediate intervention.

- To maintain 24-hour/7-day coverage of EVT, a minimum of two NI teams are required to rotate on duty.

\section{Upon Arrival at the Angiography Suite}

A customized EVT kit may be beneficial. This kit contains basic items to perform endovascular procedures, such as syringes, trays, sterile gauze, 3-way check valves, and flushing lines (Fig. 2). Considering that all items are usable during normal working hours for other endovascular procedures, a customized kit can be consumed and recreated every day or once a week, according to the expiration date of the equipment. Alternatively, a standardized stroke kit can be created and equipped in the angiography suites. This kit consists of specific devices for performing thrombectomy, such as a guide sheath, guide catheter with or without balloon, $5 \mathrm{Fr}$ angiography catheter and guidewire, rotating hemostatic valves, and aspiration catheters or stent retrievers with compatible microcatheters. A standardized stroke kit, so called BRISK (brisk recanalization ischemic stroke kit), was used in a previous report for time-saving process improvement (40). All NI team members should be well-acquainted with the

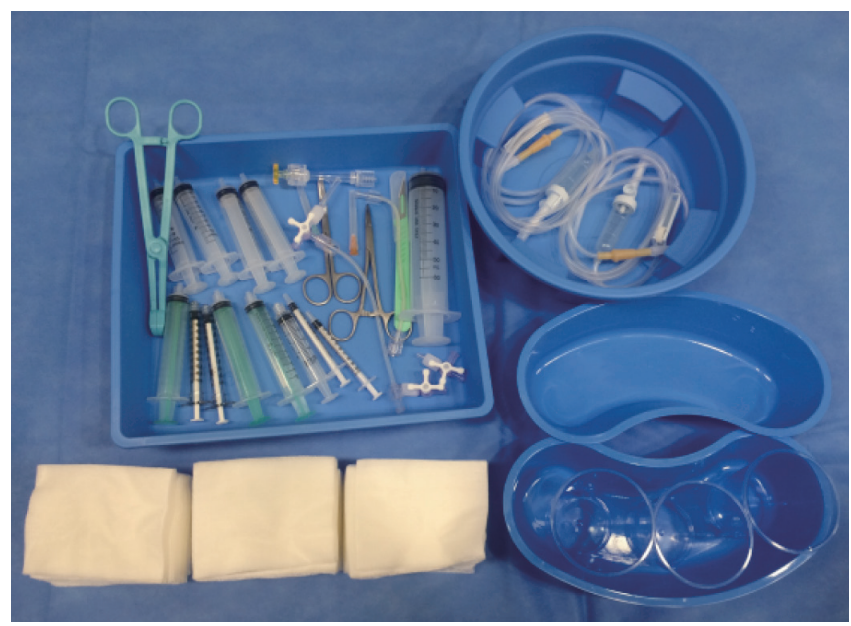

Fig. 2. Customized EVT kit. Kit contains basic equipment for endovascular procedure at discretion of NI physician, such as syringes, trays, sterile gauze, 3-way check valves, and flushing lines, which could be beneficial in reducing time for preparing procedure in offhours. $\mathrm{EVT}=$ endovascular treatment, $\mathrm{NI}=$ neurointervention exact location of the prepared EVT kit and standardized stroke kit in the angiography suite for rapid deployment. Similarly, all members should be familiar with tasks for thrombectomy procedure so that critical functions are duplicated and replaced (46).

The patient is primarily treated under local anesthesia for groin puncture during the EVT. In cases where the patient showed sustained movement or agitation, CS can be implemented. Various types of sedation have been used according to the preference of attending NI and stroke physicians. Therefore, the standardization of operator and/ or institutional protocols for CS can reduce confusion and facilitate the rapid implementation of CS. Furthermore, all NI team members should be well-informed of the standardized protocol for CS, and requisite sedatives should be equipped in the angiography suite prior to procedures. The ESCAPE trial emphasized the use of CS and less than $10 \%$ of patients were treated with general anesthesia (GA) (36). A previous retrospective multicenter study and meta-analysis reported that, compared to CS, the use of GA was associated with prolonged time to treatment, poor neurological outcomes at 90 days, and higher mortality (47, 48). Compared to GA, implementation of CS has decreased in-hospital mortality, rate of pneumonia, hospital costs, and length of stay (49). Evidence against CS for EVT include intractable patient movement during the procedure that can lead to complications, resulting in longer procedure times and delayed time to recanalization $(37,50)$. In addition, recently published two randomized controlled single-center studies demonstrated that there was no difference between CS and GA in neurological outcomes 90 days after stroke (51, 52). Although CS may allow better clinical monitoring of the neurological status and reduce treatment time, GA may benefit patients with severe agitation, vomiting, or loss of airway-protective reflexes. Finally, close monitoring of blood pressure (BP) and respiratory state should be followed by both $\mathrm{CS}$ and $\mathrm{GA}$, and establishment of standardized protocols for BP control is encouraged.

Groin puncture can be performed after standard preparation without shaving (40). If required, specialized transparent film dressing can be used when groins are not shaved. Foley catheter insertion is not mandatory prior to the procedure. In addition, it is possible to avoid intubation and arterial line placement of the patient. In previous study, $<10 \%$ of acute ischemic stroke patients required intubation for airway protection due to extreme agitation.

Besides the effort on reducing procedure time, possible 
contamination should be well controlled. Following transfemoral procedure, puncture site abscess or bacteremia rarely occurs; however, these have serious hazard potential $(53,54)$. On this ground, the best effort on infection control should be paralleled with that on reducing time.

\section{Consensus Statements}

- Customized kits for the thrombectomy should be equipped in an angiography suite to minimize delays and omission of items necessary to perform the procedure.

- Conscious sedation is recommended as the standard method in thrombectomy procedures, and formulation of a standardized protocol for CS is recommended. GA is reserved for patients for whom CS is insufficient for thrombectomy.

- Non-essential procedures such as groin shaving, Foley catheter insertion, intubation, and arterial line placement can be deferred unless indicated as an urgent or necessary procedure.

\section{Establishment of a Standardized Neurointerventional Thrombectomy Protocol}

The Mechanical Embolus Removal in Cerebral Ischemia registry have reported that the median time from groin puncture to end of procedure was 90 minutes (55). Groin puncture to first stent deployment and final digital subtraction angiography are indicators of intra-arterial procedural efficiency (56). Many factors influence the time interval between the groin puncture to final reperfusion. Patient conditions, including hemodynamic stability, respiratory state, and status of agitation or calmness can be critical variables in progression of the thrombectomy. It is imperative to build active teamwork and familiarity between the NI team members to cope with outbreak situations during the procedure. In addition, a life-saving protocol and cardiopulmonary resuscitation kit are required for managing sudden cardiac or respiratory arrest of the patient. Access issues, such as a tortuosity of the aorta and supra-aortic large arteries, are additional important factors in the delivery of treatment and subsequent reperfusion. Head and neck CTA or MRA at baseline is advantageous information to the NI physician in the planning of EVT. This includes considerations, such as selection of an appropriate catheter to overcome tortuosity of the proximal large vessel, optimal location for balloon guide catheter placement, and deployment zones for retrieval stent or an aspiration catheter (39). Tandem lesions in the proximal cervical carotid artery can be assessed simultaneously.
Although the detailed techniques and types of mechanical devices used for thrombectomy are at the discretion of the operating NI physician, standardizing the thrombectomy procedure could potentially contribute to time improvement. Considering that the experience and efficiency of the NI team may affect this metric, all NI team members are encouraged to be fully aware of the detailed sequence and relevant devices for the standardized protocol $(56,57)$. As partial recanalization is frequently obtained prior to acquisition of complete recanalization, it is difficult to determine reperfusion time accurately (41). Therefore, the metric of reperfusion time is defined as the acquisition time of > $50 \%$ of first reflow of the cerebral vascular bed beyond the lesion (58). The degree of reperfusion after the procedure should be estimated by the use of a validated metric, such as the modified Thrombolysis in Cerebral Ischemia grade (59). Finally, angiography for assessing Willisian collateral status can be deferred or omitted in most cases.

\section{Consensus Statements}

- Consistent support and active participation from all stroke team members is necessary to overcome detrimental variables of the patients.

- Acquisition of baseline vascular images, including craniocervical vasculature is strongly recommended to reduce groin puncture to first stent deployment time.

- Standardization of thrombectomy procedures may be beneficial in increasing the efficiency of the groin puncture to final reperfusion process. All NI team members should be fully aware of the detailed sequence of the protocol and relevant devices for each step in the procedure.

\section{Neurological Care after EVT}

Following EVT, the patient needs to be promptly transferred directly to the stroke or intensive care unit (24). A trained nurse in the stroke unit can provide postprocedure care for improved outcome. In cases of severe neurological deficits or unstable vital signs despite intervention, patients can be monitored in the intensive care unit. Despite successful reperfusion, paradoxical reperfusion injury may induce cerebral edema, symptomatic hemorrhagic transformation, or cerebral herniation, necessitating a careful examination of neurological changes. Risk factors for early neurological deterioration, including hyperglycemia, and inflammatory conditions such as aspiration pneumonia, should be well managed. 


\section{Consensus Statement}

- Following EVT, the patient must be transferred directly to the stroke or intensive care unit for prompt postprocedure care.

\section{Monitoring of in-Hospital Process of Care}

Monitoring of many important time indices, such as doorto-imaging, door-to-needle, imaging-to-puncture time, and feedback should be regularly provided to not only stroke and NI teams, but also to emergency physicians and nurses, CT/MR technicians, and administrative officers. This quality improvement activity allows for the early identification of workflow barriers, in addition to recognition and maintenance of best practices (2).

\section{Consensus Statements}

- Monitoring of each time metrics and feedback must be

Table 1. Workflow for Reduction of Door-To-Reperfusion Time in Acute Stroke Patients

\begin{tabular}{|c|c|}
\hline Stages & Workflow Steps \\
\hline \multirow[t]{2}{*}{ Pre-hospital phase } & Rapid pre-hospital stroke recognition with stroke screening tools \\
\hline & Pre-hospital notification and activation of stroke team \\
\hline \multirow[t]{4}{*}{ Reduction of door-to-imaging time } & Rapid notification of arrival of suspected stroke patients to the stroke team \\
\hline & "Warming call" to NI team for possible EVT \\
\hline & Direct transportation from door-to-CT scanner \\
\hline & Rapid clinical assessment and blood sampling in CT room \\
\hline \multirow[t]{4}{*}{ Reduction of imaging-to-puncture time } & No time delay of treatment by informed consent \\
\hline & Activation of NI team for EVT candidate \\
\hline & Not awaiting for response of recombinant tissue plasminogen activator treatment \\
\hline & Direct transportation from CT room to angiography suite \\
\hline \multirow[t]{4}{*}{ Reduction of puncture-to-reperfusion time } & $\begin{array}{l}\text { Deferring or eliminating non-essential procedures in favor of rapid EVT (groin shaving, } \\
\text { bladder catheter insertion, intubation, and arterial line placement) }\end{array}$ \\
\hline & Use of CS rather than general anesthesia during the procedure \\
\hline & Preparing customized kits for EVT \\
\hline & Standardization of the thrombectomy procedure \\
\hline Neurological care after EVT & Rapid admission to stroke unit and care \\
\hline Monitoring, feedback, and education & Regular provision of each time metric/quality improvement control \\
\hline
\end{tabular}

$\mathrm{CS}=$ conscious sedation, $\mathrm{CT}=$ computed tomography, EVT = endovascular treatment, NI = neurointervention

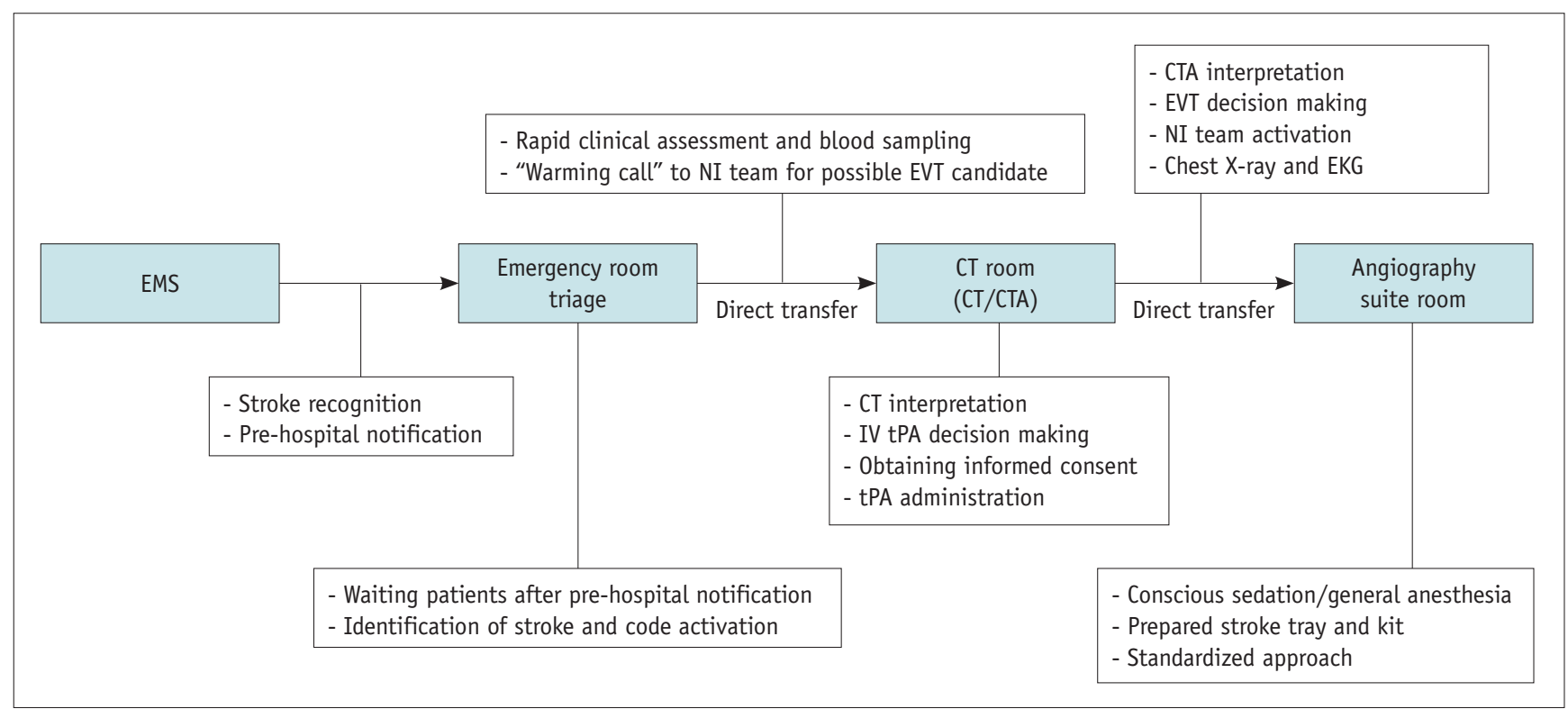

Fig. 3. Flowchart showing workflow and various steps at each stage. $C T=$ computed tomography, $C T A=C T$ angiography, $E K G=$ electrocardiography, EMS = emergency medical services 
provided to each member of the stroke and NI teams.

- A specific goal should be set for each time metric to achieve continuous quality improvement.

In summary, we present the workflow and various steps at each stage to achieve faster door-to-reperfusion time in patients treated with endovascular thrombectomy (Table 1, Fig. 3).

\section{REFERENCES}

1. Saver JL, Goyal M, van der Lugt A, Menon BK, Majoie CB, Dippel DW, et al. Time to treatment with endovascular thrombectomy and outcomes from ischemic stroke: a metaanalysis. JAMA 2016;316:1279-1288

2. Aghaebrahim A, Streib C, Rangaraju $S$, Kenmuir $C L$, Giurgiutiu DV, Horev A, et al. Streamlining door to recanalization processes in endovascular stroke therapy. J Neurointerv Surg 2017;9:340-345

3. Mehta BP, Leslie-Mazwi TM, Chandra RV, Bell DL, Sun CH, Hirsch JA, et al. Reducing door-to-puncture times for intraarterial stroke therapy: a pilot quality improvement project. $\mathrm{J}$ Am Heart Assoc 2014; 3:e000963

4. Brandler ES, Sharma M, Sinert RH, Levine SR. Prehospital stroke scales in urban environments: a systematic review. Neurology 2014;82:2241-2249

5. Kidwell CS, Starkman S, Eckstein M, Weems K, Saver JL. Identifying stroke in the field. Prospective validation of the Los Angeles prehospital stroke screen (LAPSS). Stroke 2000;31:71-76

6. Kothari RU, Pancioli A, Liu T, Brott T, Broderick J. Cincinnati prehospital stroke scale: reproducibility and validity. Ann Emerg Med 1999;33:373-378

7. Jauch EC, Saver JL, Adams HP Jr, Bruno A, Connors JJ, Demaerschalk BM, et al. Guidelines for the early management of patients with acute ischemic stroke: a guideline for healthcare professionals from the American Heart Association/ American Stroke Association. Stroke 2013;44:870-947

8. Choi B, Tsai D, McGillivray CG, Amedee C, Sarafin JA, Silver B. Hospital-directed feedback to emergency medical services improves prehospital performance. Stroke 2014;45:2137-2140

9. Wojner-Alexandrov AW, Alexandrov AV, Rodriguez D, Persse D, Grotta JC. Houston paramedic and emergency stroke treatment and outcomes study (HOPST). Stroke 2005;36:1512-1518

10. Lin CB, Peterson ED, Smith EE, Saver JL, Liang L, Xian Y, et al. Emergency medical service hospital prenotification is associated with improved evaluation and treatment of acute ischemic stroke. Circ Cardiovasc Qual Outcomes 2012;5:514522

11. Patel MD, Rose KM, O`Brien EC, Rosamond WD. Prehospital notification by emergency medical services reduces delays in stroke evaluation: findings from the North Carolina stroke care collaborative. Stroke 2011;42:2263-2268

12. Kim DH, Nah HW, Park HS, Choi JH, Kang MJ, Huh JT, et al. Impact of prehospital intervention on delay time to thrombolytic therapy in a stroke center with a systemized stroke code program. J Stroke Cerebrovasc Dis 2016;25:16651670

13. Nam HS, Park E, Heo JH. Facilitating stroke management using modern information technology. J Stroke 2013;15:135143

14. Kim SK, Lee SY, Bae HJ, Lee YS, Kim SY, Kang MJ, et al. Prehospital notification reduced the door-to-needle time for IV t-PA in acute ischaemic stroke. Eur J Neurol 2009;16:13311335

15. Lin CB, Peterson ED, Smith EE, Saver JL, Liang $L$, Xian $Y$, et al. Patterns, predictors, variations, and temporal trends in emergency medical service hospital prenotification for acute ischemic stroke. J Am Heart Assoc 2012;1:e002345

16. Hong KS, Bang OY, Kim JS, Heo JH, Yu KH, Bae HJ, et al. Stroke statistics in Korea: part II stroke awareness and acute stroke care, a report from the Korean Stroke Society and Clinical Research Center for Stroke. J Stroke 2013;15:67-77

17. Heo JH, Kim YD, Nam HS, Hong KS, Ahn SH, Cho HJ, et al. A computerized in-hospital alert system for thrombolysis in acute stroke. Stroke 2010;41:1978-1983

18. Kansagra AP, Meyers GC, Kruzich MS, Cross DT 3rd, Moran CJ. Wide variability in prethrombectomy workflow practices in the united states: a multicenter survey. AJNR Am J Neuroradiol 2017;38:2238-2242

19. Heldner MR, Zubler C, Mattle HP, Schroth G, Weck A, Mono $M L$, et al. National institutes of health stroke scale score and vessel occlusion in 2152 patients with acute ischemic stroke. Stroke 2013;44:1153-1157

20. Rajajee V, Kidwell C, Starkman S, Ovbiagele B, Alger JR, Villablanca P, et al. Early MRI and outcomes of untreated patients with mild or improving ischemic stroke. Neurology 2006;67:980-984

21. Wang H, Thevathasan A, Dowling R, Bush S, Mitchell P, Yan B. Streamlining workflow for endovascular mechanical thrombectomy: lessons learned from a comprehensive stroke center. J Stroke Cerebrovasc Dis 2017;26:1655-1662

22. Schrock JW, Lum M. Drill down analysis of door-to-needle time of acute ischemic stroke patients treated with intravenous tissue plasminogen activator. Am J Emerg Med 2014;32:13301333

23. Zerna C, Assis Z, d'Esterre CD, Menon BK, Goyal M. Imaging, intervention, and workflow in acute ischemic stroke: the calgary approach. AJNR Am J Neuroradiol 2016;37:978-984

24. Goyal M, Menon BK, Hill MD, Demchuk A. Consistently achieving computed tomography to endovascular recanalization < 90 minutes: solutions and innovations. Stroke 2014; 45:e252-e256

25. Lev MH, Farkas J, Rodriguez VR, Schwamm LH, Hunter GJ, Putman CM, et al. Ct angiography in the rapid triage of patients with hyperacute stroke to intraarterial thrombolysis: accuracy in the detection of large vessel thrombus. J Comput Assist Tomogr 2001;25:520-528

26. Powers WJ, Derdeyn CP, Biller J, Coffey CS, Hoh BL, Jauch 
EC, et al. 2015 American Heart Association/American Stroke Association focused update of the 2013 guidelines for the early management of patients with acute ischemic stroke regarding endovascular treatment: a guideline for healthcare professionals from the American Heart Association/American Stroke Association. Stroke 2015;46:3020-3035

27. Casaubon LK, Boulanger JM, Blacquiere D, Boucher S, Brown K, Goddard T, et al. Canadian stroke best practice recommendations: hyperacute stroke care guidelines, update 2015. Int J Stroke 2015;10:924-940

28. Lee JS, Demchuk AM. Choosing a hyperacute stroke imaging protocol for proper patient selection and time efficient endovascular treatment: lessons from recent trials. J Stroke 2015;17:221-228

29. Brinjikji W, Demchuk AM, Murad MH, Rabinstein AA, McDonald RJ, McDonald JS, et al. Neurons over nephrons: systematic review and meta-analysis of contrast-induced nephropathy in patients with acute stroke. Stroke 2017;48:1862-1868

30. Sharma J, Nanda A, Jung RS, Mehta S, Pooria J, Hsu DP. Risk of contrast-induced nephropathy in patients undergoing endovascular treatment of acute ischemic stroke. J Neurointerv Surg 2013;5:543-545

31. Tu JV, Willison DJ, Silver FL, Fang J, Richards JA, Laupacis A, et al. Impracticability of informed consent in the Registry of the Canadian Stroke Network. N Engl J Med 2004;350:14141421

32. Korean law for emergency medical services. Web site. http://www.law.go.kr/lsInfoP.do?lsiSeq=199337\&ef $\mathrm{Yd}=20171203 \# 0000$. Updated 2017. Accessed January 15, 2018

33. Ribo M, Molina CA, Cobo E, Cerda N, Tomasello A, Quesada H, et al. Association between time to reperfusion and outcome is primarily driven by the time from imaging to reperfusion. Stroke 2016;47:999-1004

34. Sun CH, Nogueira RG, Glenn BA, Connelly K, Zimmermann $\mathrm{S}$, Anda K, et al. "Picture to puncture": a novel time metric to enhance outcomes in patients transferred for endovascular reperfusion in acute ischemic stroke. Circulation 2013;127:1139-1148

35. Menon BK, Sajobi TT, Zhang Y, Rempel JL, Shuaib A, Thornton $\mathrm{J}$, et al. Analysis of workflow and time to treatment on thrombectomy outcome in the endovascular treatment for small core and proximal occlusion ischemic stroke (ESCAPE) randomized, controlled trial. Circulation 2016;133:2279-2286

36. Goyal M, Demchuk AM, Menon BK, Eesa M, Rempel JL, Thornton J, et al. Randomized assessment of rapid endovascular treatment of ischemic stroke. $N$ Engl J Med 2015;372:1019-1030

37. Menon BK, Almekhlafi MA, Pereira VM, Gralla J, Bonafe A, Davalos A, et al. Optimal workflow and process-based performance measures for endovascular therapy in acute ischemic stroke: analysis of the solitaire FR thrombectomy for acute revascularization study. Stroke 2014;45:2024-2029

38. Qureshi AI, Egila H, Adil MM, Siddiqi H, Mian N, Hassan AE, et al. "No turn back approach" to reduce treatment time for endovascular treatment of acute ischemic stroke. J Stroke Cerebrovasc Dis 2014;23:e317-e323

39. Menon BK, Demchuk AM. Computed tomography angiography in the assessment of patients with stroke/TIA. Neurohospitalist 2011;1:187-199

40. Frei D, McGraw C, McCarthy K, Whaley M, Bellon RJ, Loy D, et al. A standardized neurointerventional thrombectomy protocol leads to faster recanalization times. J Neurointerv Surg 2017;9:1035-1040

41. Leifer D, Bravata DM, Connors JJ 3rd, Hinchey JA, Jauch $\mathrm{EC}$, Johnston SC, et al. Metrics for measuring quality of care in comprehensive stroke centers: detailed followup to brain attack coalition comprehensive stroke center recommendations: a statement for healthcare professionals from the American Heart Association/American Stroke Association. Stroke 2011;42:849-877

42. Almekhlafi MA, Hockley A, Desai JA, Nambiar V, Mishra S, Volny 0 , et al. Overcoming the evening/weekend effects on time delays and outcomes of endovascular stroke therapy: the Calgary Stroke Program experience. J Neurointerv Surg 2014;6:729-732

43. Rai AT, Smith MS, Boo S, Tarabishy AR, Hobbs GR, Carpenter JS. The 'pit-crew' model for improving door-to-needle times in endovascular stroke therapy: a Six-Sigma project. J Neurointerv Surg 2016;8:447-452

44. Tai YJ, Weir L, Hand P, Davis S, Yan B. Does a 'code stroke' rapid access protocol decrease door-to-needle time for thrombolysis? Intern Med J 2012;42:1316-1324

45. Barba R, Losa JE, Velasco M, Guijarro C, Garcia de Casasola G, Zapatero A. Mortality among adult patients admitted to the hospital on weekends. Eur J Intern Med 2006;17:322-324

46. McTaggart RA, Ansari SA, Goyal M, Abruzzo TA, Albani B, Arthur $A J$, et al. Initial hospital management of patients with emergent large vessel occlusion (ELVO): report of the standards and guidelines committee of the society of neurointerventional surgery. J Neurointerv Surg 2017;9:316323

47. Abou-Chebl A, Lin R, Hussain MS, Jovin TG, Levy EI, Liebeskind DS, et al. Conscious sedation versus general anesthesia during endovascular therapy for acute anterior circulation stroke preliminary results from a retrospective, multicenter study. Stroke 2010;41:1175-1179

48. Brinjikji W, Murad MH, Rabinstein AA, Cloft HJ, Lanzino G, Kallmes DF. Conscious sedation versus general anesthesia during endovascular acute ischemic stroke treatment: a systematic review and meta-analysis. AJNR Am J Neuroradiol 2015;36:525-529

49. McDonald JS, Brinjikji W, Rabinstein AA, Cloft HJ, Lanzino $G$, Kallmes DF. Conscious sedation versus general anaesthesia during mechanical thrombectomy for stroke: a propensity score analysis. J Neurointerv Surg 2015;7:789-794

50. Schönenberger S, Uhlmann L, Hacke W, Schieber S, Mundiyanapurath S, Purrucker JC, et al. Effect of conscious sedation vs general anesthesia on early neurological improvement among patients with ischemic stroke undergoing 
endovascular thrombectomy: a randomized clinical trial. JAMA 2016;316:1986-1996

51. Löwhagen Hendén $P$, Rentzos A, Karlsson JE, Rosengren L, Leiram B, Sundeman H, et al. General anesthesia versus conscious sedation for endovascular treatment of acute ischemic stroke: the AnStroke trial (anesthesia during stroke). Stroke 2017;48:1601-1607

52. Schregel K, Behme D, Tsogkas I, Knauth M, Maier I, Karch $A$, et al. Effects of workflow optimization in endovascularly treated stroke patients - a pre-post effectiveness study. PLoS One 2016;11:e0169192

53. Samore MH, Wessolossky MA, Lewis SM, Shubrooks SJ $\mathrm{Jr}$, Karchmer AW. Frequency, risk factors, and outcome for bacteremia after percutaneous transluminal coronary angioplasty. Am J Cardiol 1997;79:873-877

54. Culver DA, Chua J, Rehm SJ, Whitlow P, Hertzer NR. Arterial infection and staphylococcus aureus bacteremia after transfemoral cannulation for percutaneous carotid angioplasty and stenting. J Vasc Surg 2002;35:576-579

55. Sun CH, Ribo M, Goyal M, Yoo AJ, Jovin T, Cronin CA, et al. Door-to-puncture: a practical metric for capturing and enhancing system processes associated with endovascular stroke care, preliminary results from the rapid reperfusion registry. J Am Heart Assoc 2014;3:e000859

56. Eesa M, Menon BK, Hill MD, Demchuk A, Goyal M. Achieving faster recanalization times by IA thrombolysis in acute ischemic stroke: where should we direct our efforts? Interv Neuroradiol 2011;17:228-234

57. Eesa M, Almekhlafi MA, Mitha AP, Wong JH, Goyal M. Manual aspiration thrombectomy through balloon-tipped guide catheter for rapid clot burden reduction in endovascular therapy for ICA L/T occlusion. Neuroradiology 2012;54:12611265

58. Berkhemer OA, Fransen PS, Beumer D, van den Berg LA, Lingsma $\mathrm{HF}, \mathrm{Yoo} A J$, et al. A randomized trial of intraarterial treatment for acute ischemic stroke. $N$ Engl J Med 2015;372:11-20

59. Higashida RT, Furlan AJ, Roberts H, Tomsick T, Connors B, Barr J, et al. Trial design and reporting standards for intraarterial cerebral thrombolysis for acute ischemic stroke. Stroke 2003;34:e109-e137 